\title{
Improvement in Clinical Outcomes, Right Ventricular Function and Pulmonary Artery Hypertensions after Half Dose Thrombolysis in Patients with High Risk Sub-Massive Pulmonary Thromboembolism
}

\author{
Ramalingam Vadivelu1, Nagendra Boopathy Senguttuvan², Ravindran Rajendran ${ }^{3}$, Kathiresan Jeyashree ${ }^{4}$, \\ Anand Subramanian ${ }^{5}$, Vinod Kumar B. ${ }^{6}$, Ramesh S. ${ }^{7}$, Muralidharan T. R. ${ }^{8}$ \\ ${ }^{1}$ Assistant Professor, Department of Cardiology, Velammal Medical College and Hospital, \\ Madurai, Tamilnadu, India. \\ ${ }^{2}$ Assistant Professor, Department of Cardiology, Sri Ramachandra Institute of Higher Education and Research, \\ Chennai, Tamilnadu, India. \\ ${ }^{3}$ Assistant Professor, Department of Cardiology, Trichy SRM Medical College Hospital and Research Centre, \\ Trichy, Tamilnadu, India. \\ ${ }^{4}$ Assistant Professor, Department of Community Medicine, Velammal Medical College and Hospital, \\ Madurai, Tamilnadu, India. \\ ${ }^{5}$ Consultant Interventional Cardiologist, Department of Cardiology, Kauvery Hospitals, \\ Trichy, Tamilnadu, India. \\ ${ }^{6}$ Assistant Professor, Department of Cardiology- Sri Ramachandra Institute of Higher Education and Research, \\ Chennai, Tamilnadu, India. \\ ${ }^{7}$ Assistant Professor, Department of Cardiology- Sri Ramachandra Institute of Higher Education and Research, \\ Chennai, Tamilnadu, India. \\ ${ }^{8}$ HOD, Department of Cardiology, Sri Ramachandra Institute of Higher Education and Research, \\ Chennai, Tamilnadu, India.
}

\section{ABSTRACT}

\section{BACKGROUND}

Role of fibrinolysis in sub-massive pulmonary thromboembolism (PTE) is not clear. Improved hemodynamics with increased bleeding with fibrinolysis in patients with high risk sub massive PTE when compared to anticoagulation alone without mortality benefit is known.

\section{METHODS}

We retrospectively studied the effect of low dose fibrinolysis in patients with submassive pulmonary thromboembolism in four centers over a period of two years. Submassive PTE is defined as major PTE with RV dysfunction and myocardial injury (elevated troponin) but without cardiogenic shock. Baseline prethrombolysis assessment of clinical parameters and hemodynamics of the patient, RV function assessment with TAPSE, RV Fractional Area Change (FAC) and Pulmonary Artery Pressure (PAP) were assessed and all the readings were repeated post thrombolysis.

\section{RESULTS}

A total of 123 patients was screened, out of which, 72 patients (58\%) had sub massive pulmonary thromboembolism. Out of 72 patients, $11(15 \%)$ patients who had been treated with low dose fibrinolysis for varied reasons were included in the study. Mean \pm SD age of the study population was $58.36 \pm 10.20$ years with $54.5 \%$ of them being males. Deep vein thrombosis was a predisposing factor in 7 patients (63\%).

\section{CONCLUSIONS}

LDF is feasible, effective and safe in patients with high risk submassive PTE who had at least intermediate risk for bleeding. In almost all patients except in one, RV function improved, and PA pressure reduced significantly. We propose that a similar tailored approach in patients with PTE may improve the clinical outcomes with better safety profile.

\section{KEY WORDS}

Pulmonary Thromboembolism, Fibrinolysis, Pulmonary Artery Hypertension, RV Function
Corresponding Author:

Dr. Nagendra Boopathy Senguttuvan, Assistant Professor and Senior Consultant Cardiology, Sri Ramachandra Institute of Higher Education and Research, Chennai-600116, Tamilnadu, India. E-mail: drsnboopathy@gmail.com

DOI: $10.14260 /$ jemds/2019/583

Financial or Other Competing Interests: None.

How to Cite This Article:

Vadivelu R, Senguttuvan NB, Rajendran $R$, et al. Improvement in clinical outcomes, right ventricular function and pulmonary artery hypertensions after half dose thrombolysis in patients with high risk submassive pulmonary thromboembolism. J. Evolution Med. Dent. Sci. 2019;8(34): 2681-2686,DOI:

$10.14260 /$ jemds $/ 2019 / 583$

Submission 05-07-2019, Peer Review 11-08-2019,

Acceptance 17-08-2019,

Published 26-08-2019.

\section{(i) $(3)$}




\section{BACKGROUND}

Acute myocardial infarction, stroke and pulmonary thromboembolism (PTE) constitute the three major cardiovascular problems with significant mortality and morbidity. PTE and deep venous thrombosis are clubbed together to constitute venous thromboembolism (VTE). PTE remains one of the most common causes for sudden cardiac death.(1) Hence, it should be suspected in any patient with new onset dyspnoea, chest pain, palpitations, syncope or unexplained hypotension.(1,2),(3) It was estimated that prevalence of PTE was 1\% among patients hospitalized in a general hospital.(4) Major cardiovascular risk factors associated with coronary artery disease were also associated with PTE.(5) An autopsy based Indian study(6) showed that $15.9 \%$ of the 100 patients in their autopsy series had pulmonary thromboembolism of whom, it was fatal or contributory to death in almost $78 \%$ of patients. Sepsis, hepatobiliary pancreatic diseases, cardiovascular diseases and malignancy were the leading causes underlying the problem.(6) Major morbidities associated with VTE include recurrent VTE, post thrombotic syndrome, and chronic thromboembolic pulmonary hypertension (CTEPH). Based on the clinical presentation, patients with PTE are categorized into massive, sub-massive and low risk PTE.(1) Patients with low systolic BP $<90 \mathrm{mmHg}$ are categorized as massive PTE. Those with evidence of right ventricular dysfunction documented by either echocardiogram or CT with preserved systolic BP are categorized into sub-massive PTE, while those who have no RV dysfunction with normal BP are low risk individuals. Case fatality rate of VTE varies from $1 \%$ to $60 \%$ depending the particular clinical presentation.(1) Mortality rate for massive PTE and sub-massive PTE were 58.3\% and $15.1 \%$ as demonstrated by the International Cooperative Pulmonary Embolism Registry (ICOPER). Though most major societal guidelines and statements agree with the management of massive and low risk PTE in the form of fibrinolysis along with heparin therapy and heparin alone respectively, there exists a significant discordant amongst them in the management of sub-massive PTE.(7-10) Hence the role of fibrinolysis in sub-massive pulmonary thromboembolism (PTE) is not clear. It is associated with improved hemodynamics with increased bleeding without improvement in survival.

Only one large trial adequately powered to assess clinical outcomes had shown improvement in hemodynamics with reduction of pulmonary artery pressure (PAP) with fibrinolysis(11) in patients with high risk sub massive PTE when compared to anticoagulation alone. Still it is a common clinical practice to administer fibrinolytic agents in all patients with sub-massive PTE. Bleeding associated with any anti-thrombotic agent is directly proportional to the dosage administered. Evidence for low dose fibrinolysis (LDF) is not robust. Role of LDF in patients with submassive PTE is not well studied.(12) We aimed to study the efficacy and safety of reduced dose fibrinolytic agents in patients with high risk PTE.

\section{METHODS}

We retrospectively studied the effect of LDF in patients with sub massive PTE in four centers over a period of two years. The studied centres include the respective departments of cardiology at Sri Ramachandra Institute of Higher Education and Research, Chennai, Velammal Medical College Hospital and Research Institute, Madurai, Chennai Medical College and Hospital, Trichy and Kauvery Hospitals, Trichy. Medical records of patients who had been diagnosed with pulmonary thromboembolism was initially screened. It is an investigator initiated, non-funded study.

\section{Study Population}

We defined sub-massive PTE as patients who have had recent onset of symptoms ( $<15$ days) with CT pulmonary angiogram proven or radionuclide proven diagnosis of PTE. In addition, they should also have right ventricular dilatation proven by either echocardiogram or CT scan along with myocardial injury documented by elevated cardiac troponins but without cardiogenic shock. Only patients who had received nonconventional (Lower doses of fibrinolytic agents) for varied reasons were included as study participants. Informed consent was obtained from all the patients. Institute ethical committee clearance was also obtained for the study. Baseline characteristics of the included patients were studied along with all available laboratory parameters that included electrocardiogram, transthoracic echocardiogram and CTpulmonary angiogram. Nature of fibrinolytic agent administered, and oral anticoagulants started by the treating physicians, were also recorded. Baseline pre thrombolysis assessment of clinical parameters and hemodynamics of the patient, RV function assessment with TAPSE, RV Fractional area change (FAC) and pulmonary artery pressure (PAP) were assessed. All readings were repeated post thrombolysis. Primary outcome of the study was to study the efficacy of lower doses of fibrinolytic agents and their safety. Outcomes that were studied included death, hemodynamic decompensation that required addition of inotropic agent(s) or addition of extra doses of fibrinolytic agent. Safety assessment included documentation of major and minor bleedings as described by bleeding and academic consortium (BARC). ${ }^{13}$

\section{Statistical Methods}

Data was extracted from case sheets and investigations sheets and entered into Microsoft Excel. IBM SPSS v20 was used to analyse the data. The clinical parameters before and after thrombolysis were compared using chi square test and paired $\mathrm{t}$ test as appropriate. A p value $<0.05$ was taken as significant.

\section{RESULTS}

A total of eleven patients with submassive PTE who received low dose thrombolysis were retrospectively studied. The clinical, demographic and bleeding risk profile of the study population is described in Table 1 . Mean \pm SD age of the study population was $58.36 \pm 10.20$ years and $54.5 \%$ were males. Average duration of symptoms was $6.5 \pm 8.5$ days and mean 
duration since acute worsening was $23.5 \pm 18.0$ hours. Almost all patients had dyspnoea with NYHA Class II in $18.2 \%$, NYHA Class III in $27.3 \%$ and NYHA class IV in $54.5 \%$ of the patients. Hypertension and diabetes mellitus were prevalent in $5(45.5 \%)$ patients and 2 patients $(18.2 \%)$ were obese. History of recent surgery was present in 2 patients (18.2\%), among whom one patient developed PTE in the first post-op day. History of trauma was present in 2 patients (18.2\%) and recent immobilization was seen in 3 patients $(27.3 \%)$. History of long travel was present in 1 patient (9.1\%).

The mean HAS-BLED score of the study population was 2.18. Only one patient was provided low dose fibrinolytic agent in spite of low HAS-BLED score due to frailty of the individual. The most commonly used fibrinolytic agent in the study was alteplase [6 patients (54.6\%)] followed by Tenecteplase [4 patients (36.4\%)]. Streptokinase was used in one patient $(9.1 \%)$. Regarding the parenteral anticoagulant use, unfractionated heparin was used in 7 patients $(63.6 \%)$, low molecular weight heparin was used in 3 patients $(27.3 \%)$ and fondaparinux in one patient $(9.1 \%)$. At discharge, newer oral anti-coagulants (NOACS) were the most commonly used oral anticoagulants [Apixaban (3 patients $\{27.3 \%\}$ ); Rivaroxaban (1 patient $\{9.1 \%\}$ ); Dabigatran (4 patients $\{36.4 \%\})]$. Warfarin was used in 2 patients $(18.2 \%)$ and Acitrom in one patient $(9.1 \%)$

Characteristics of Pulmonary Artery Involvement and Pulmonary Embolism Severity Index (PESI)

CT angiography demonstrated the distribution of thrombus according to the pulmonary artery involvement as follows: Left MPA in 4 patients (40\%), Right MPA in 5 patients (50\%), both RPA and LPA in 4 patients (40\%). All of the study subjects had submassive PTE and majority of them were in class II [4 patients (36.4\%)] and class IV PESI [3 (27.3\%)] as shown in Table 2.

\section{Comparison of Clinical and Echocardiographic Parameters Before and After Thrombolysis}

The important clinical and vital echocardiographic parameters changes were observed before and after fibrinolysis (Table 3) Among the clinical parameters compared before and after half dose thrombolysis, the significant difference was observed in pulse rate (PR) with mean PR reducing from $114.09 \pm 12.300 \mathrm{bpm}$ to $89.64 \pm$ $14.521 \mathrm{bpm}, \mathrm{p}<0.001$. Respiratory rate reduced from $27.73 \pm$ 4.819 per minute to $17.82 \pm 4.535, \mathrm{p}<0.001$. Oxygen saturation improved from $90.09 \pm 5.7 \%$ to $95.7273 \pm 2.3 \%$, $\mathrm{p}=0.003$ and $\mathrm{PaO}_{2}$ increased from $57.55 \pm 5.067$ to $75.45 \pm$ $7.230 \mathrm{mmHg}, \mathrm{p}<0.001$. Peak pulmonary artery pressure dramatically reduced from $71.00 \pm 18.341 \mathrm{mmHg}$ to $40.36 \pm$ $18.554 \mathrm{mmHg},<0.001$. Also, there is improvement in $\mathrm{RV}$ function in the form of increase of TAPSE from $11.45 \pm 1.440$ to $17.27 \pm 2.687 \mathrm{~mm}, \mathrm{p}<0.001$ and significant increase of RV functional area change (FAC) from $29.18 \pm 2.714 \%$ to $39.55 \pm$ $5.184 \%, \mathrm{p}<0.001$.

\section{Outcomes After Low Dose Fibrinolysis (Table 4)}

No in- hospital mortality or mortality at 6 months follow up was observed in the study. Mean duration of hospital stay was $7.64 \pm 3.695$ days. Out of 11 patients, 10 patients had successful reduced dose thrombolysis and thrombolysis failed in one patient. In that one patient in whom fibrinolysis failed tenecteplase was given. Failure was documented by the presence of persistence of tachycardia and echocardiogram showing no improvement in RV function and PA pressure. Repeat half dose thrombolysis done after 6 hours of initial failed half dose thrombolysis did not significantly improve the clinical, hemodynamic and echocardiographic parameters in that patient. He had persistent RV dysfunction and severe pulmonary artery hypertension. He also required one repeat hospitalisation for right heart failure. At 6 months of follow up, he was alive and class II and had eventually developed chronic severe pulmonary thromboembolic PAH. Major bleeding occurred in one patient in the form of postoperative site bleed from hysterectomy site requiring blood transfusion. She was a morbidly obese patient who developed submassive PTE on the first post op day after hysterectomy. Upon initiating low dose thrombolysis, she responded clinically and improved but on the second day had major bleeding with $\mathrm{Hb}$ drop requiring blood transfusion.'

\begin{tabular}{|c|c|}
\hline Demographic Profile & \\
\hline Age $($ Mean \pm SD) & $58.36 \pm 10.20$ \\
\hline \multirow[t]{2}{*}{ SEX - no. (\%) } & Male - 6 (54.5\%) \\
\hline & Female - $5(45.5 \%)$ \\
\hline Duration of symptoms (days) & $6.5 \pm 8.5$ \\
\hline Acute worsening since (hours) & $23.5 \pm 18.0$ \\
\hline \multicolumn{2}{|l|}{ Symptomatology } \\
\hline \multirow[t]{3}{*}{ Dyspnoea } & Class 2- $2(18.2 \%)$ \\
\hline & Class $3-3(27.3 \%)$ \\
\hline & Class 4 - $6(54.5 \%)$ \\
\hline Syncope- no.\% & $1(9.1 \%)$ \\
\hline Chest Pain & $5(45.5 \%)$ \\
\hline Cough & $4(36.4 \%)$ \\
\hline Haemoptysis & $1(9.1 \%)$ \\
\hline Palpitations & $6(54.5 \%)$ \\
\hline \multicolumn{2}{|l|}{ Medical History } \\
\hline Deep Vein Thrombosis & $\begin{array}{c}7 \text { (70\%) [Right iliac vein-1(9.1\%); Right femoral- } \\
1(9.1 \%) ; \text { left femoral -1(9.1\%); right popliteal - } \\
\text { 2(27.3\%); left popliteal-2(18.2\%); }\end{array}$ \\
\hline Diabetes Mellitus & $5(45.5 \%)$ \\
\hline Malignancy & $1(9.1 \%)$ \\
\hline Hypertension & $5(45.5 \%)$ \\
\hline Recent surgery & $2(18.2 \%)$ \\
\hline Pregnancy & $0(0 \%)$ \\
\hline Chronic kidney disease & $1(9.1 \%)$ \\
\hline COPD & $1(9.1 \%)$ \\
\hline Recent immobilisation & $3(27.3 \%)$ \\
\hline Trauma & $2(18.2 \%)$ \\
\hline Long Travel & $1(9.1 \%)$ \\
\hline Obesity & $2(18.2 \%)$ \\
\hline \multicolumn{2}{|l|}{ HAS-BLED Score } \\
\hline 0 & $1(9.1 \%)$ \\
\hline 1 & $2(18.2 \%)$ \\
\hline 2 & $5(45.5 \%)$ \\
\hline 3 & $1(9.1 \%)$ \\
\hline 4 & $1(9.1 \%)$ \\
\hline 5 & $1(9.1 \%)$ \\
\hline \multicolumn{2}{|c|}{ Table 1. Baseline Characteristics of the Study Population } \\
\hline
\end{tabular}

\begin{tabular}{|c|c|}
\hline Pulmonary Artery Involvement & \\
\hline Left main PA- no. (\%) & $4(40 \%)$ \\
\hline Right main PA- no. (\%) & $5(50 \%)$ \\
\hline Both RPA and LPA & $4(40 \%)$ \\
\hline \multicolumn{2}{|l|}{ PTE Severity } \\
\hline Sub massive & $11(100 \%)$ \\
\hline \multirow{5}{*}{ PES Index - no. (\%) } & Class I - 1 (9.1\%) \\
\hline & Class II- $4(36.4 \%)$ \\
\hline & Class III- $1(9.1 \%)$ \\
\hline & Class IV- $3(27.3 \%)$ \\
\hline & Class V- $2(18.2 \%)$ \\
\hline $\begin{array}{r}\text { Table 2. Pulmonary Artery } \\
\text { Charac }\end{array}$ & nt and PTE Severity \\
\hline
\end{tabular}




\begin{tabular}{|c|c|c|c|}
\hline $\begin{array}{c}\text { Clinical } \\
\text { Parameters }\end{array}$ & $\begin{array}{c}\text { Before } \\
\text { Thrombolysis } \\
\text { Mean } \pm \text { SD }\end{array}$ & $\begin{array}{c}\text { After Thrombolysis } \\
\text { Mean ( } \pm \text { SD) }\end{array}$ & p Value \\
\hline Pulse Rate (bpm) & $114.09 \pm 12.300$ & $89.64 \pm 14.521$ & $<0.001$ \\
\hline Systolic BP (mmHg) & $112.73 \pm 9.045$ & $116.18 \pm 12.376$ & 0.240 \\
\hline Diastolic BP (mmHg) & $71.82 \pm 9.81650$ & $73.8182 \pm 7.87170$ & 0.629 \\
\hline Respiratory rate (/min) & $27.73 \pm 4.819$ & $17.82 \pm 4.535$ & $<0.001$ \\
\hline Oxygen saturation & $90.09 \pm 5.70008$ & $95.7273 \pm 2.32770$ & 0.003 \\
\hline $\mathrm{PaO}_{2}(\mathrm{mmHg})$ & $57.55 \pm 5.067$ & $75.45 \pm 7.230$ & $<0.001$ \\
\hline $\begin{array}{c}\text { Peak Pulmonary artery } \\
\text { pressure (mmHg) }\end{array}$ & $71.00 \pm 18.341$ & $40.36 \pm 18.554$ & $<0.001$ \\
\hline TAPSE (mm) & $11.45 \pm 1.440$ & $17.27 \pm 2.687$ & $<0.001$ \\
\hline RV FAC (\%) & $29.18 \pm 2.714$ & $39.55 \pm 5.184$ & $<0.001$ \\
\hline Table 3. Clinical and Echocardiographic Parameters before and after \\
Thrombolysis \\
\hline \multicolumn{4}{|c|}{} \\
\hline
\end{tabular}

\begin{tabular}{|c|c|}
\hline Death- no. (\%) & 0 \\
\hline Major bleeding- no. (\%) & $1(9.1 \%)$ \\
\hline Need for surgery for bleeding- no. (\%) & 0 \\
\hline Need for Blood transfusion - no. (\%) & $1(9.1 \%)$ \\
\hline Minor bleeding - no. (\%) & 0 \\
\hline $\begin{array}{c}\text { Improvement in RV dysfunction - no. (\%) } \\
\text { Improvement in Pulmonary artery } \\
\text { hypertension - no. (\%) }\end{array}$ & $10(90.9 \%)$ \\
\hline $\begin{array}{c}\text { Persistent Pulmonary hypertension- no. } \\
(\%)\end{array}$ & $10(90.9 \%)$ \\
\hline Successful thrombolysis - no. (\%) & $1(9.1 \%)$ \\
\hline Failed thrombolysis - no. (\%) & $10(90.9 \%)$ \\
\hline Duration of stay - no of days \pm SD & $1(9.1 \%)$ \\
\hline Repeat hospitalisation- no. (\%) & $7.64 \pm 3.695$ \\
\hline \multicolumn{2}{|c|}{ Table 4. Outcomes after Half Dose Thrombolysis } \\
\hline \multicolumn{2}{|c|}{}
\end{tabular}
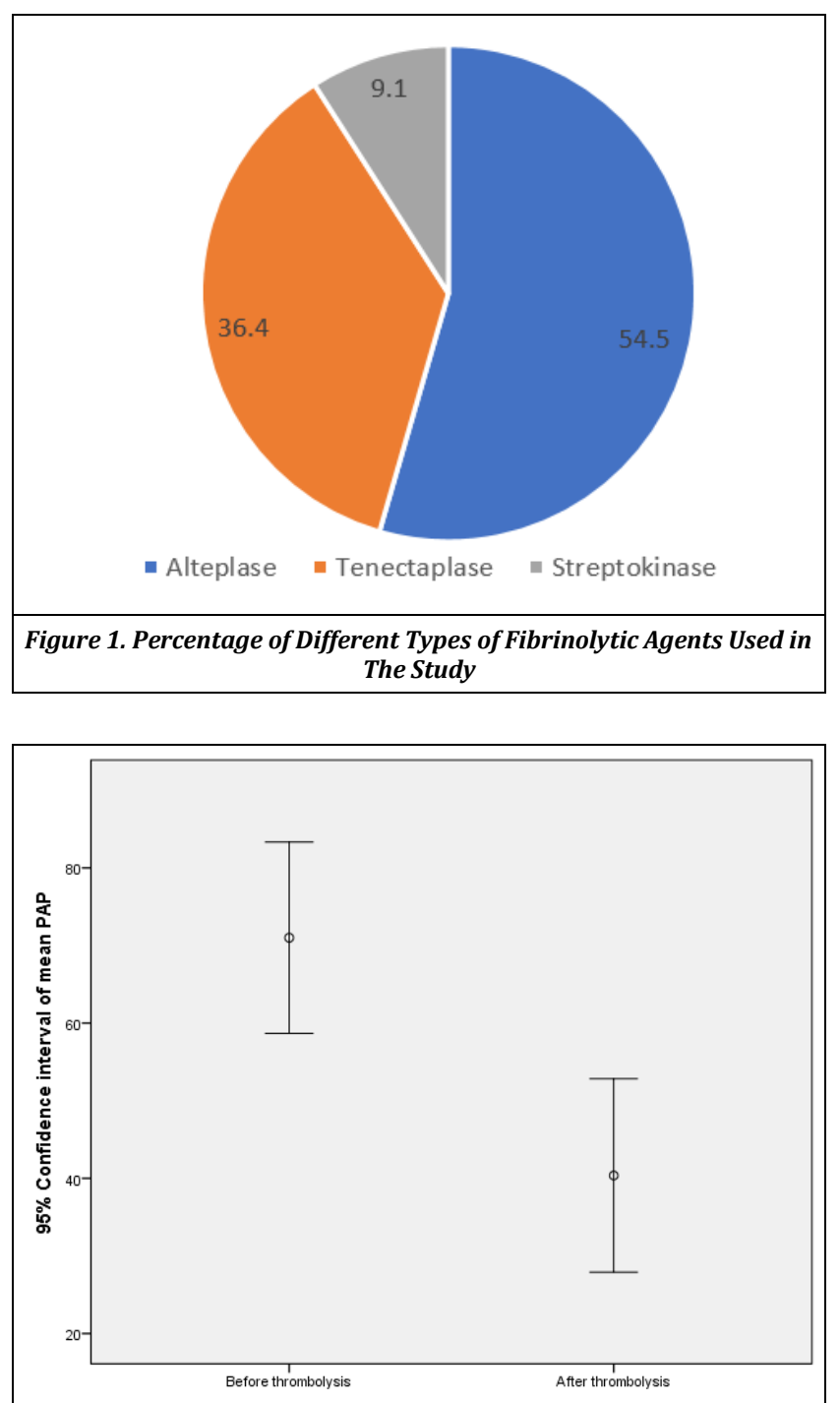

Figure 2. Error Bar Graph Showing Distribution of Mean Systolic Pulmonary Artery Pressure before and after Low Dose Fibrinolysis. There is Significant Reduction of PAP after LDF.

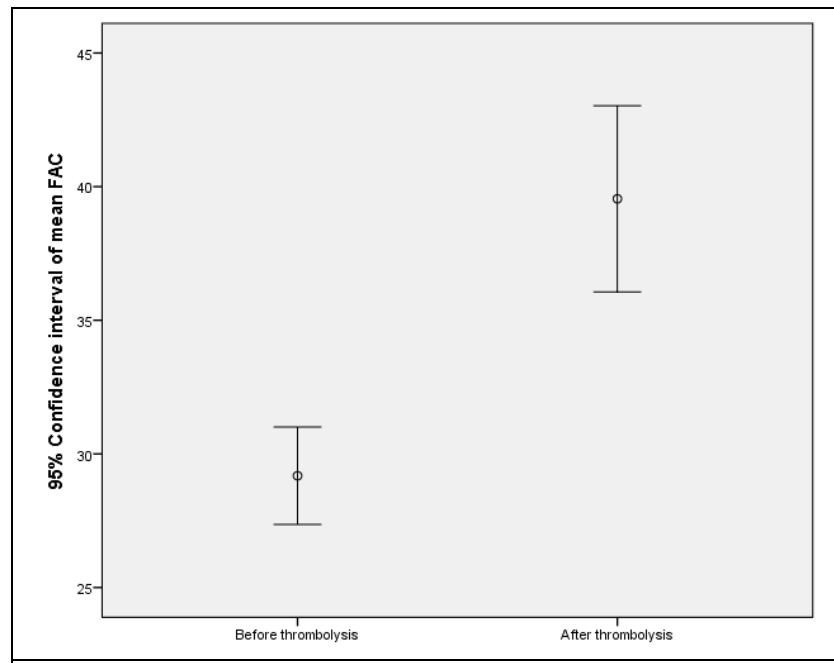

Figure 3. Error Bar Graph Showing Distribution of Mean Right Ventricular Fractional Area Change before and after Low Dose Fibrinolysis. There is Significant Improvement of FAC after LDF.

\section{DISCUSSION}

We observed that provision of low dose fibrinolysis is effective in most of the patients with high risk submassive PTE who had intermediate risk for bleeding. It resulted in significant improvement in hemodynamics in the form of reduction in pulmonary artery hypertension and improvement in RV function.

Current evidence suggests that there is no clear benefit for thrombolysis over heparin anticoagulation in patients with acute sub-massive PTE unlike massive PTE where fibrinolytic therapy is associated with significant reduction in mortality, pulmonary hypertension and recurrent PTE. However, in patients with submassive PTE, the standard of treatment is not clear, ${ }^{(7-10)}$ as anticoagulation therapy may be associated with suboptimal recanalization of pulmonary artery and clinical outcomes. Fibrinolytic therapy results in better recanalization and reduction of PAP and improves RV function but at the cost of increased bleeding risk. Hence to achieve better recanalization and RV function with reduced bleeding risk reduced dose fibrinolysis might be a viable option.

Role of fibrinolysis in hemodynamically stable PTE was first studied by Goldhaber et al.(14) They found significant improvement in right-ventricular wall motion and decrease in RV end diastolic area at 24 hours patients who received alteplase and heparin as compared of patients who received heparin alone. They also observed significant absolute improvement in pulmonary perfusion in patients receiving alteplase and heparin. There were 2 fatal and 3 non-fatal clinically suspected recurrent PEs were noticed within 14 days in those who received alone. Four out of 46 patients (8.7\%) had major bleeding (One non-fatal intracranial bleed; 3 major bleeds requiring blood transfusion) whereas in heparin alone group only one patient $(1.8 \%)$ had minor bleed. Hence the study concluded that fibrinolysis using alteplase resulted in rapid improvement of right ventricular function and pulmonary perfusion with possible lower rate of net adverse clinical outcomes.

PEITHO trial(11) analysed the outcomes of fibrinolysis with tenecteplase vs heparin therapy in patients with 
intermediate risk pulmonary embolism. The composite end point of death or hemodynamic decompensation within the first 7 days after randomization with standard anticoagulation was $5.6 \%$ whereas it was only $2.6 \%$ with fibrinolytic therapy which was very significant. But this benefit was overcome by significant increase in extracranial and intracranial bleeding with a $2.0 \%$ rate of haemorrhagic stroke and a $6.3 \%$ rate of major extracranial haemorrhage which was significantly higher when compared with heparin group $(0.2 \%$ rate of haemorrhagic stroke and a $1.2 \%$ rate of major extracranial haemorrhage). There was no significant difference in the mortality end point between two groups (2.4\% with TG vs $3.2 \%$ in the heparin group, $\mathrm{p}=0.42$ ). In our study one patient had developed a operative site bleeding from hysterectomy wound on post op day 1 when she developed submassive PTE and was treated with half dose thrombolysis. She required blood transfusion for correcting the haematocrit otherwise no surgical intervention required.

As lung receives $100 \%$ of cardiac output unlike brain or heart, dosage of the same may not be same in PTE as compared to ischemic stroke or MI. Previously two studies have addressed this issue of efficacy and safety of reduced dose of fibrinolysis in patients with PTE with massive PTE and moderate PTE respectively.(12,15) Study has addressed this question by using half dose alteplase in patients with moderate PTE. MOPETT(12) trial investigators showed half dose thrombolysis is more effective in patients with intermediate risk PTE at 28 months when compared to anticoagulation alone. Around $16 \%$ of the patients ( 9 of 58 patients) in thrombolysis group (TG) developed Pulmonary hypertension $(\mathrm{PAH})$ whereas in anticoagulation group 57\% ( 32 of 56 patients) developed $\mathrm{PAH},(\mathrm{p}<0.001)$. The composite end point of PAH and recurrent PTE developed in 9 of 58 patients $(16 \%)$ in the TG and 35 of 56 patients $(63 \%)$ in the CG ( $\mathrm{p}<0.001)$, respectively. Surprisingly none of the patients in either of the arms developed any bleeding. The duration of hospitalization was shorter in TG $(2.2 \pm 0.5$ days in the TG and $4.9 \pm 0.8$ days in the CG), ( $<<0.001)$. There was no significant difference when mortality and recurrent PTE was assessed as a combined endpoint [1 (1.6\%) in TG and 6 $(10 \%$ ) in the CG], (p 0.0489). There was no significant difference in the rate of individual outcomes of death and recurrent PE when assessed independently also.

In our study also its clearly evident that $90.9 \%$ of the patients ( 9 out of 11 patients) had remarkable reduction in PA pressures (Mean peak PA pressure reduced from 71.00 $\pm 18.341 \mathrm{mmHg}$ to $40.36 \pm 18.554 \mathrm{mmHg}$ ) after thrombolysis. RV function improved significantly in $90.9 \%$ of the patients which was objectively shown in the form of improvement in TAPSE $(11.45 \pm 1.440$ to $17.27 \pm 2.687, \mathrm{p}<0.001)$ and RV FAC $(29.18 \pm 2.714 \%$ to $39.55 \pm 5.184 \%,<0.001)$ after thrombolysis. A large retrospective analysis recently found no benefit of providing half dose fibrinolysis and it had similar mortality and rates of major bleeding as compared to full dose therapy with increased treatment escalation happening more often in half-dose-treated patients.(16) Two metanalysis addressing the role of thrombolysis are available after PEITHO. $(17,18)$ One of them ${ }^{(17)}$ included all patients with PTE and found that thrombolytic therapy reduced all-cause mortality with an increased risks of ICH and major bleeding. In their sub group analysis, they found the same effect in patients with hemodynamically stable PTE with RV dysfunction. While the second study that included patients with hemodynamically stable alone concluded that, thrombolysis should not be used for most normotensive PE patients as it increased risk for major bleeding and intra cranial hemorrhage without any evidence of reduction in mortality (18). This could be due to difference in the statistical methodology and inclusion of patients with stable hemodynamics alone in the second group. In our study 10 out of 11 patients (90.9\%) showed improvement in RV function after thrombolysis. One patient had persistent pulmonary artery hypertension and RV dysfunction despite initial low dose thrombolysis followed by full dose thrombolysis. At follow up 6 months duration he had developed chronic PTE related severe $\mathrm{PAH}$.

Though not well studied definite screening tool is available to determine the bleeding risk of an individual after fibrinolysis, few studies have addressed this issue and found elderly people with higher diastolic BP, higher BMI and associated invasive procedures were associated with major bleeding.(19) In patients who undergo percutaneous coronary interventions ( $\mathrm{PCI})$, ischemia and bleeding risk are currently calculated to determine the duration of dual anti platelets regimen. We propose such a similar tailored approach in patients with PTE may improve the clinical outcomes with better safety profile.

\section{Limitations}

It was a retrospective, descriptive study. Hence the limitations applicable to all non-randomized, observational studies without a control group are applicable to our study too. Sample size of our study is also small. Different types of fibrinolytic agents used in our study also adds to the heterogeneity. Despite these limitations, we strongly make the case for conducting an adequately powered, randomized controlled trial for assessing the efficacy and safety of low dose fibrinolysis in patients with high sub-massive PTE as compared to full dose and heparin alone arms. Though not standardized, we have HAS-BLED score to assess the bleeding risk in the absence of a well validated score for the same purpose.

\section{CONCLUSIONS}

Our study suggests that low dose fibrinolysis is effective and safe in patients with high risk sub-massive PTE who had intermediate risk for bleeding with significant reduction in pulmonary artery hypertension and improvement in RV function and clinical outcomes. No hemodynamic decompensation or intracranial bleeding was noticed. No deaths or recurrent PTE were noted.

\section{REFERENCES}

[1] Agnelli G, Becattini C. Acute pulmonary embolism. N Engl J Med 2010;363(3):266-74.

[2] Chunilal SD, Eikelboom JW, Attia J, et al. Does this patient have pulmonary embolism? JAMA 2003;290(21):284958. 
[3] Prandoni P, Lensing AWA, Prins MH, et al. Prevalence of pulmonary embolism among patients hospitalized for syncope. N Engl J Med 2016;375(16):1524-31.

[4] Stein PD, Henry JW. Prevalence of acute pulmonary embolism among patients in a general hospital and at autopsy. Chest 1995;108(4):978-81.

[5] Gregson J, Kaptoge S, Bolton T, et al. Cardiovascular risk factors associated with venous thromboembolism. JAMA Cardiol 2019;4(2):163-73.

[6] Kakkar N, Vasishta RK. Pulmonary embolism in medical patients: an autopsy-based study. Clin Appl Thromb Hemost 2008;14(2):159-67.

[7] Konstantinides SV, Torbicki A, Agnelli G, et al. 2014 ESC guidelines on the diagnosis and management of acute pulmonary embolism. Eur Heart J 2014;35(43):3033-69, 3069a-3069k.

[8] Jaff MR, McMurtry MS, Archer SL, et al. Management of massive and submassive pulmonary embolism, iliofemoral deep vein thrombosis, and chronic thromboembolic pulmonary hypertension: a scientific statement from the American Heart Association. Circulation 2011;123(16):1788-830.

[9] Fesmire FM, Brown MD, Espinosa JA, et al. Critical issues in the evaluation and management of adult patients presenting to the emergency department with suspected pulmonary embolism. Ann Emerg Med 2011;57(6):62852.e75.

[10] Damlo S. AAFP and ACP Publish Recommendations on Diagnosis and Management of VTE. Am Fam Physician 2007;76(8):1225-9.

[11] Meyer G, Vicaut E, Danays T, et al. Fibrinolysis for patients with intermediate-risk pulmonary embolism. N Engl J Med 2014;370(15):1402-11.
[12] Sharifi M, Bay C, Skrocki L, et al. “MOPETT” Investigators. Moderate pulmonary embolism treated with thrombolysis (from the "MOPETT" Trial). Am J Cardiol 2013;111(2):273-7.

[13] Mehran R, Rao SV, Bhatt DL, et al. Standardized bleeding definitions for cardiovascular clinical trials: a consensus report from the Bleeding Academic Research Consortium. Circulation 2011;123(23):2736-47.

[14] Goldhaber SZ, Haire WD, Feldstein ML, et al. Alteplase versus heparin in acute pulmonary embolism: randomized trial assessing right-ventricular function and pulmonary perfusion. Lancet 1993;341(8844):50711.

[15] Wang C, Zhai Z, Yang Y, et al. Efficacy and safety of low dose recombinant tissue-type plasminogen activator for the treatment of acute pulmonary thromboembolism: a randomized, multicenter, controlled trial. Chest 2010;137(2):254-62.

[16] Kiser TH, Burnham EL, Clark B, et al. Half-dose versus full-dose alteplase for treatment of pulmonary embolism. Crit Care Med 2018;46(10):1617-25.

[17] Chatterjee S, Chakraborty A, Weinberg I, et al. Thrombolysis for pulmonary embolism and risk of allcause mortality, major bleeding, and intracranial hemorrhage: a meta-analysis. JAMA 2014;311(23):241421.

[18] Riera-Mestre A, Becattini C, Giustozzi M, et al. Thrombolysis in hemodynamically stable patients with acute pulmonary embolism: a meta-analysis. Thromb Res 2014;134(6):1265-71.

[19] Meyer G, Gisselbrecht M, Diehl JL, et al. Incidence and predictors of major hemorrhagic complications from thrombolytic therapy in patients with massive pulmonary embolism. Am J Med 1998;105(6):472-7. 